\title{
RELIEF RESTORATION OF COMPLICATED FORM OBJECTS BY MONOCHROMATIC MICROWAVE RADIATION
}

\author{
Ivan Kuzmenko ${ }^{1,1}$, Vladimir Yakubov ${ }^{1}$, Timur Muksunov ${ }^{2}$ \\ ${ }^{1}$ Tomsk State University, 634050, Tomsk, Russia \\ ${ }^{2}$ V. D. Kuznetsov Siberian Physical-Technical Institute at Tomsk State University, 634050, Tomsk, \\ Russia
}

\begin{abstract}
Article demonstrates possibility of monochromatic radiation usage for relief restoration. There is a problem with restoration when scanned object is not flat and it is not parallel to the scanning plane. It was discovered that two-dimensional phase distribution could be applied for distance determination. It is reliable way to solve problems listed above. In conclusion offered methods allow monochromatic microwave radiation usage for screening system development.
\end{abstract}

\section{Actuality of problem}

Public places' safety assurance is complicated problem which actuality is increasing every year. Terrorism is a large treat for many countries including Russian Federation.

Important infrastructural objects, such as airports, equipped with wide range of technical instruments purposed for human and luggage inspection. Traditional way of search system engineering based on x-ray scanning. But x-ray radiation can be dangerous for human health, especially after multiple exposing. This fact is affirmed by existence of such parameter as number of allowed scans per year. Because of that, already long time researches of alternative ways ago have been started. The alternative scan systems should be as effective as X-ray and safe for human health as well [1-5].

Radiowave scan systems were invented recently. They have image quality at the same level with x-ray introscope and safe for health - allowed number of scans per year is not limited. But existing radiowave scan systems are expensive, costing millions of USD [6, 7].

One of the reasons of such big price is high costing for UHF transceivers with wide frequency range. Adapting of monochromatic radiation is possible way to lower scan system price. Figure 1 presents view of microwave sensor array.

\footnotetext{
${ }^{1}$ Corresponding author: kuzmenko.ivan.yu@gmail.com
} 


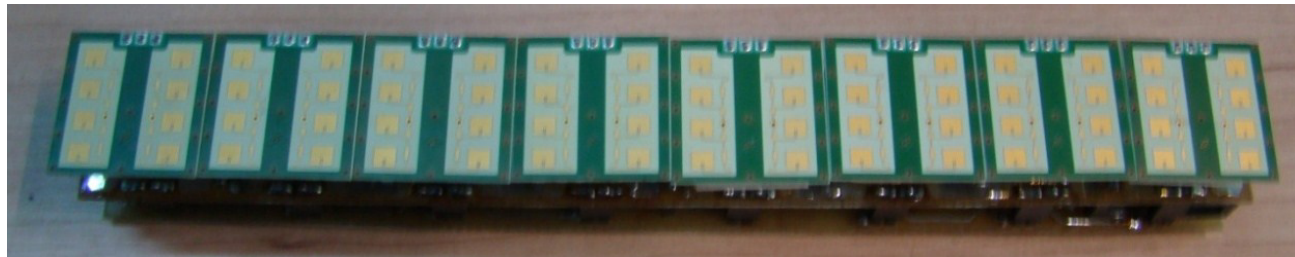

Figure 1. RSM 2650 sensor array.

Certain results of such researching had already achieved. Figure 2 presents testing object and its radio image which was restored by aperture synthesis method after scanning with sensor array demonstrated above.

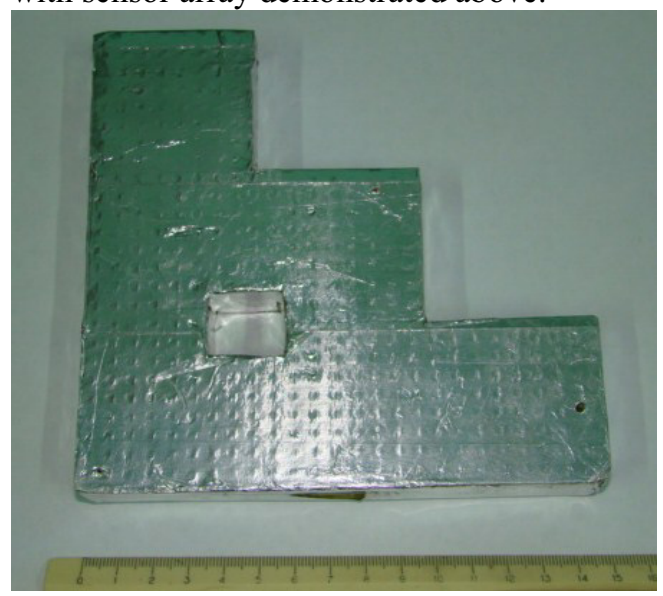

a)

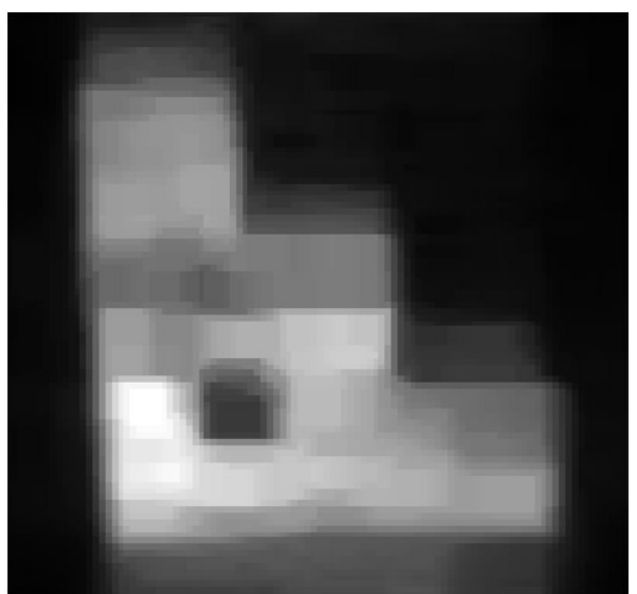

b)

Figure 2. Testing object (a) and its radioimage (b).

Restored radioimage shows possibility of radiowave scanning system with monochrome sensors. But during the experiment distance of testing object was known before scanning and apparatus system function (ASF) was calculated for exactly that length. In real situation distance can be unknown. In addition to that, objects of scanning, such as human body, usually have non-flat relief.

\section{Mathematic modeling}

It's necessary to define how will change interferential image when object is not oriented in parallel to scanning plane. For this purpose such situation was modeled with metal pipe as scanning object. Pipe axis is angled by $10^{\circ}$ to scanning plane. Interferential images of the pipe in cases of parallel and angled orientation are shown by Figure 3. According to the image, angled orientation causes interferential lines to bend. Restored radioimages are shown by Figure 4. The radioimage of inclined pipe is blurred because of unfocused radiation. 


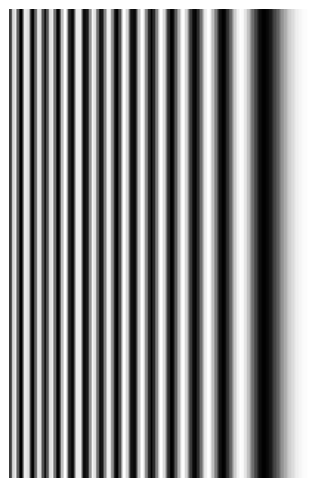

a)

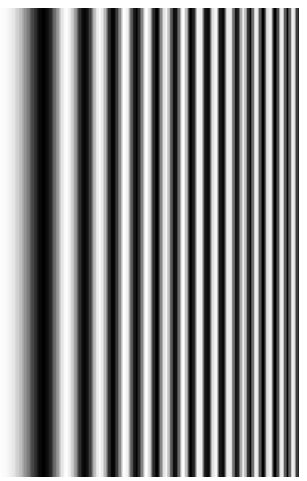

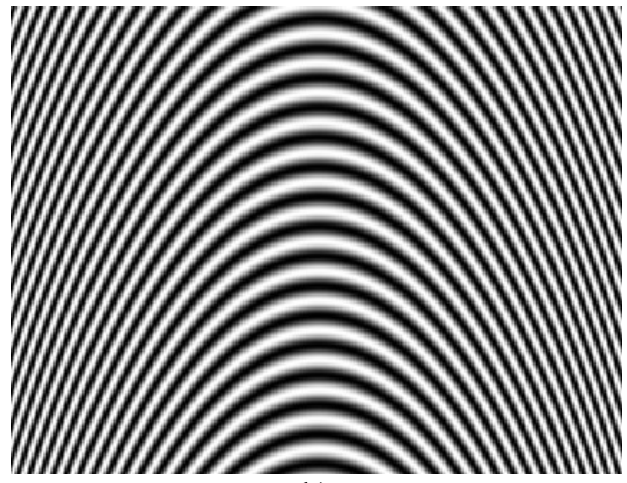

b)

Figure 3. Interferential image of metal pipe with parallel (a) and angled (b) orientation to scanning plane.

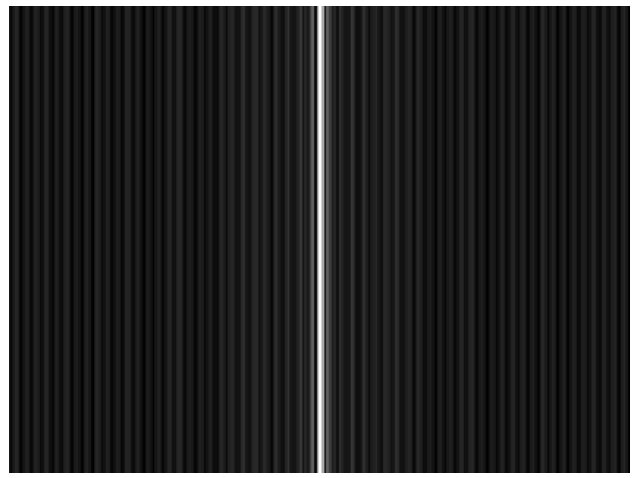

a)

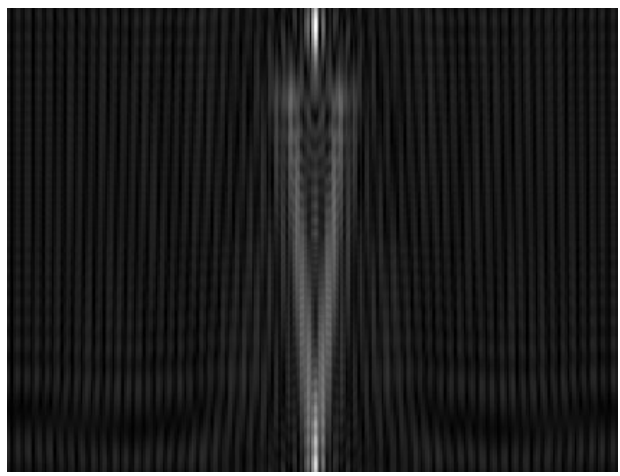

б)

Figure 4. Radioimage of metal pipe with parallel (a) and angled (b) orientation to scanning plane.

It will be shown next how changes radioimage in case of ASF for radiation received is calculated for wrong distance.

Figure 5 shows that in this case radioimage quality is considerably reduced. Object positioning is still correct, but its size is considerably bigger than the true one.

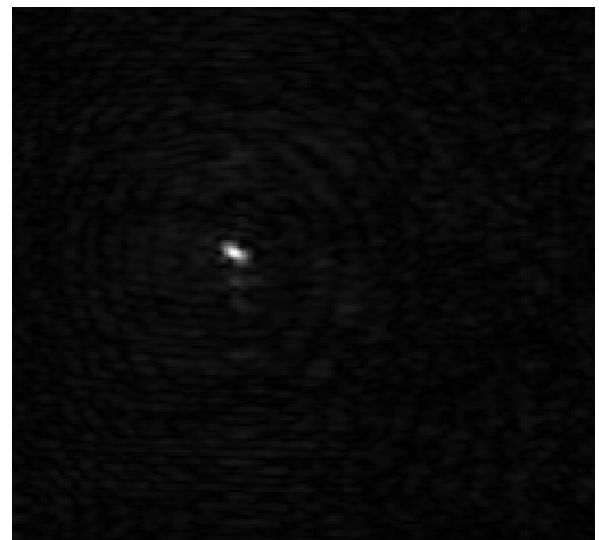

a)

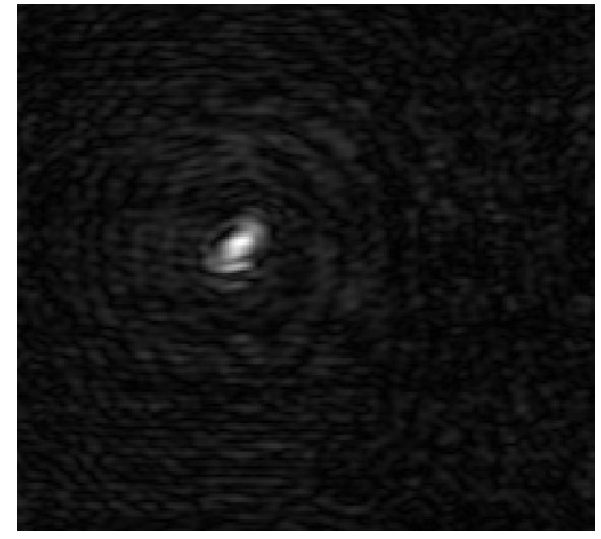

b)

Figure 5. Radioimage in cases of ASF focusing on true (a) and error (b) distance. 


\section{Experimental distance definition and relief restoration}

It is known fact that it is possible to define distance by multipositional scanning even in case of monochrome oscillations as scanning signal. Research results of distance definition possibility in case of Doppler sensor array multipositional scanning are presented below.

Figure 6, $a$ shows received signal after multipositional scanning of metal pipe at $15 \mathrm{~cm}$ distance from scanning plane. For distance definition the signal was focused on distance from $0.1 \mathrm{~cm}$ to $30 \mathrm{~cm}$ with $0.1 \mathrm{~cm}$ step. "Focusing signal on distance" means operation of convolution between ASF calculated for that distance and signal received during scanning. Result is presented at Figure 6, $b$. Image shows that there is distinguishable maximum at 15 $\mathrm{cm}$ distance which is corresponding to the object location. This result proofs that monochrome multipositional scanning allows definition of object distance with adequate precision. $\mathrm{cm}$

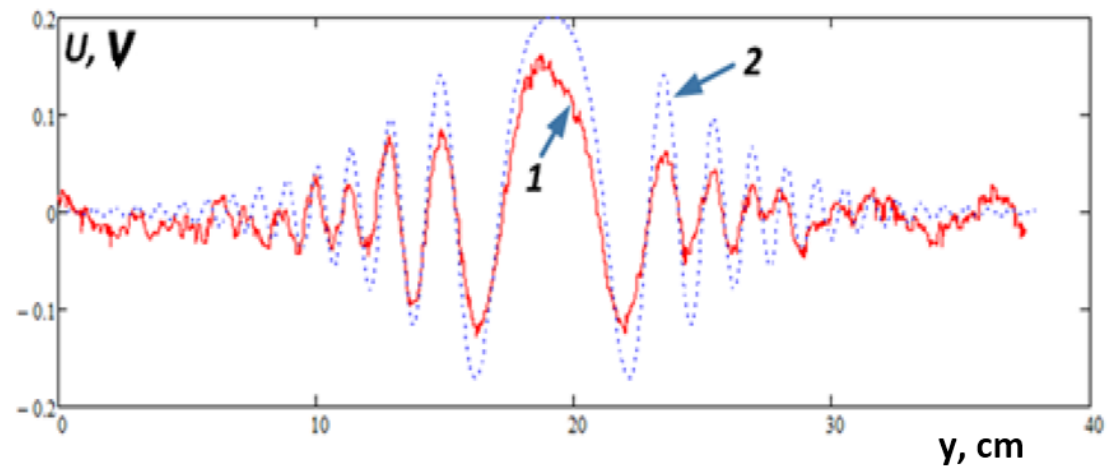

a)

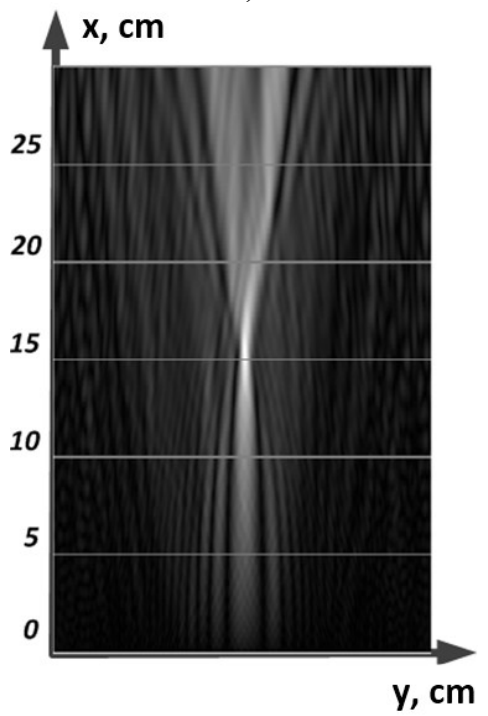

b)

Figure 6. Signal received during multipositional scanning (a) and results of focusing on various distances (b).

Application of radiowave scanner is searching for forbidden items covered on human body under clothing. Relief of human body is not flat, it has its own protrusions and depressions. 
Applying to the radioimage restoration for such difficult object it means necessity of distance definition for proper radiation focusing.

Serial of experiments was done to solve this problem. One of such experiments consisted of radioimage restoration of two metal plates with $2 \mathrm{~mm}$ distance shift between. Plates had size of $35 \times 15 \mathrm{~cm}$ and their photograph is presented at Figure 7, $a$. Figure 7, $b$ shows result of interferential image focusing on nearest plate distance. But it is not impossible to define which one is nearest by using the image. Focusing on another plate also does not allow correct relief restoration.

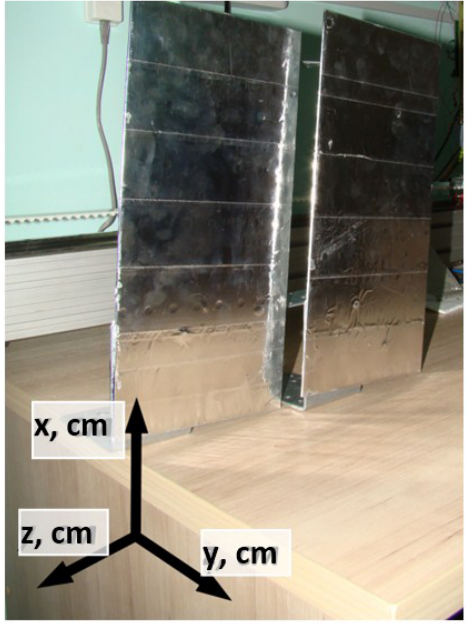

a)

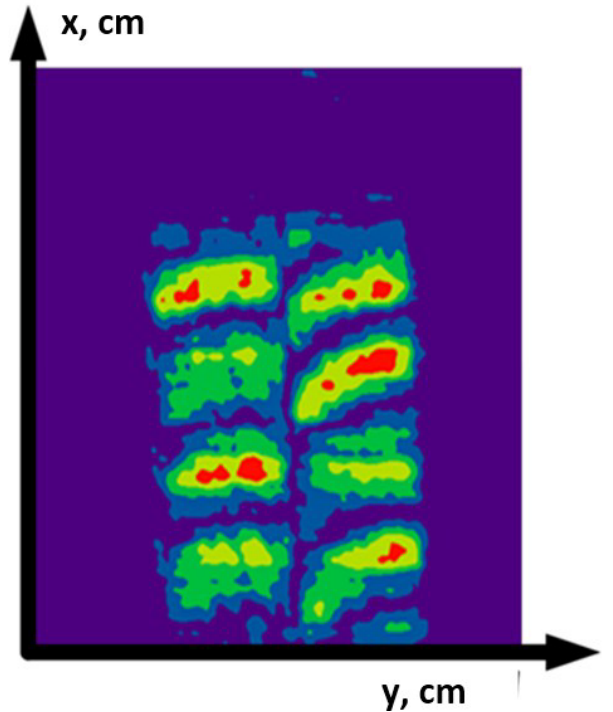

b)

Figure 7. Testing object photograph (a) and it's radioimage (b).

Phase information of radioimage can be used for relief restoration of scanned object. Line 2 at Figure 8 shows dimensional phase distribution at horizontal axis of scanned object. Line 1 shows intensity of the signal. As the graph demonstrates, phase of the signal along the single plane stays on the same level and makes distinguishable leap at the border between plates.

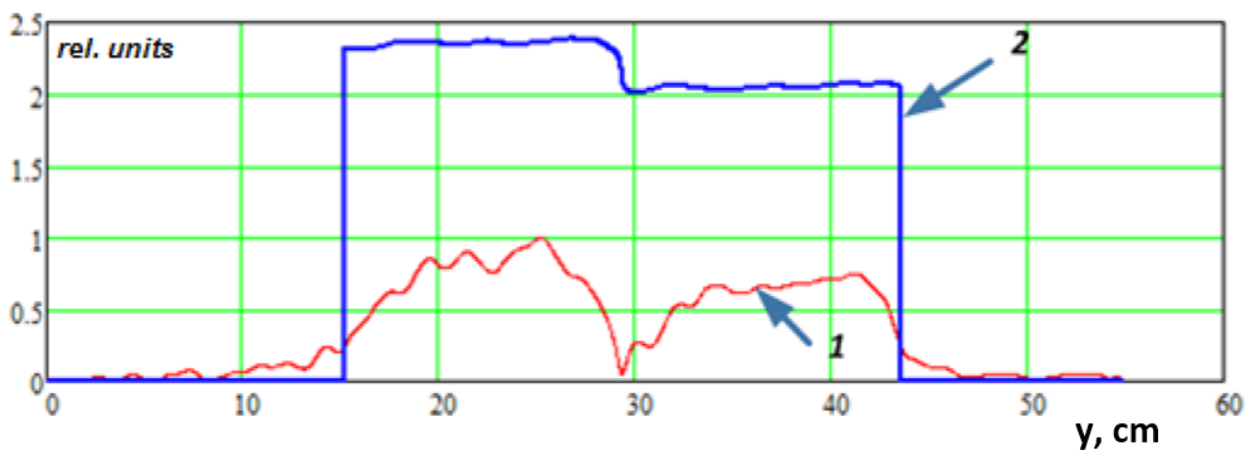

Figure 8. Dimensional phase distribution.

Testing object for next experiment was made of metal plate and dielectric brick glued together. Photograph of the object is shown by Figure 9,a. Metal plate is trapezoid with 9 
$\mathrm{cm}$ and $16 \mathrm{~cm}$ bottoms and $25 \mathrm{~cm}$ height. Size of dielectric brick is $2 \times 5 \times 9 \mathrm{~cm}$. Interferential image is shown by Figure 9, $b$, restored radioimage is shown by Figure 9, c. Form of metal plate is defined and presence of brick is detected.

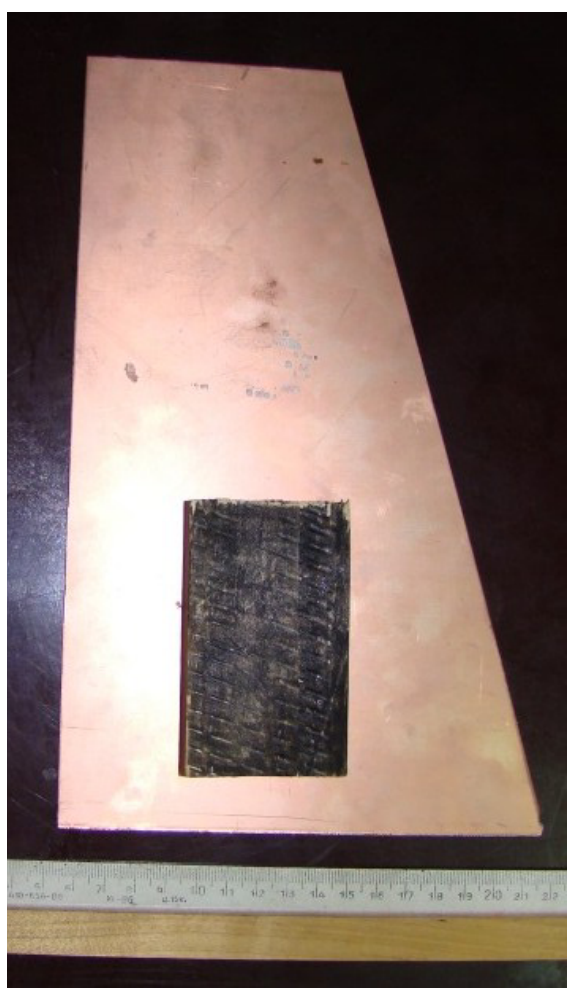

a)

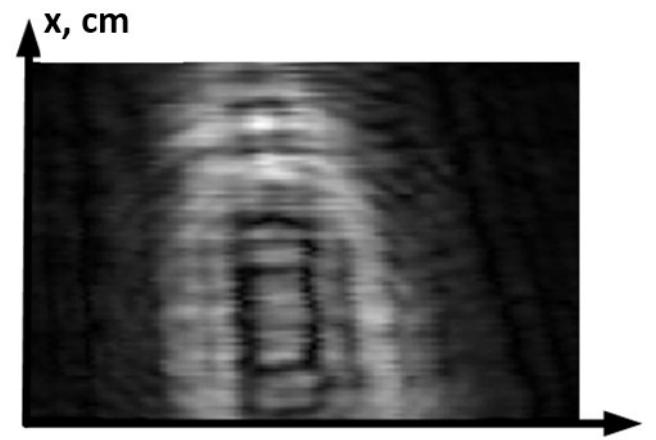

y, cm

b)

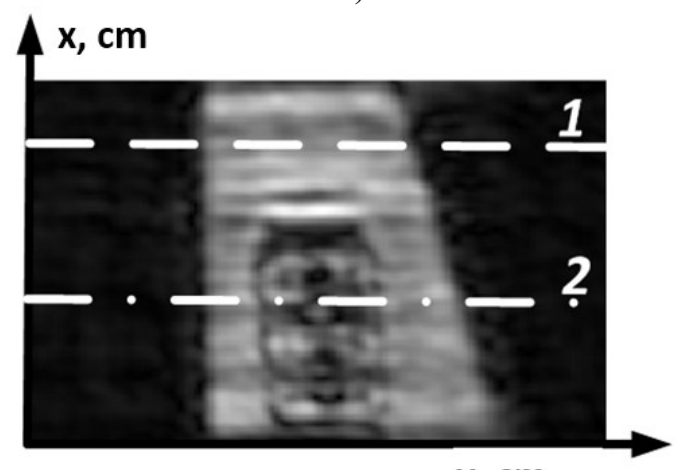

y, cm

c)

Figure 9. Testing object photograph (a), interferential image (b) and radioimage (c).

Two strings of the image were chosen for research. Intensity and phase distribution along line 1 from Figure 9, $c$ are shown by Figure 10, a graph. As it shows, image phase along metal plate stays at nearly same level. Intensity and phase distribution along line 2 from Figure 9, $c$ are shown by Figure 10, $b$ graph. As it shows, image phase makes a leap at the dielectric brick position. 


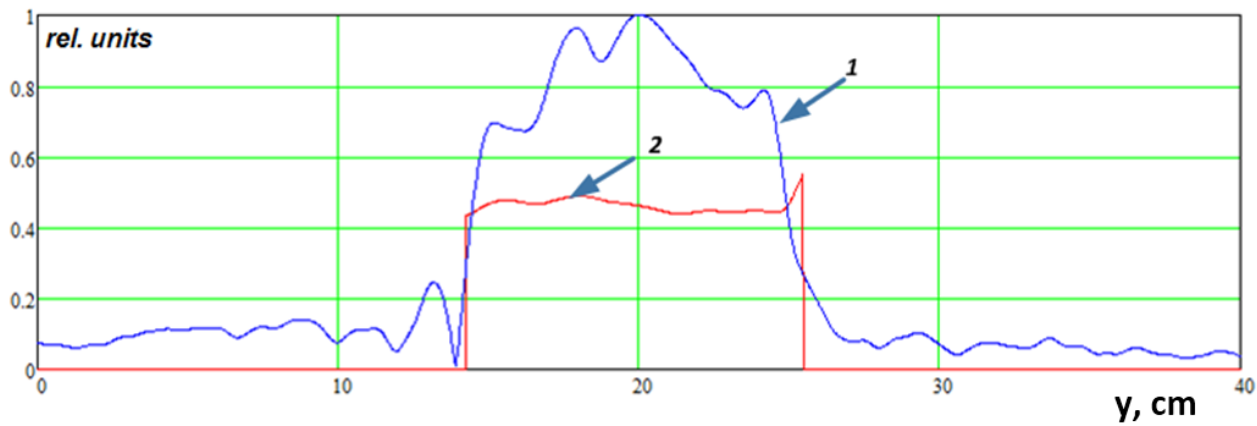

a)

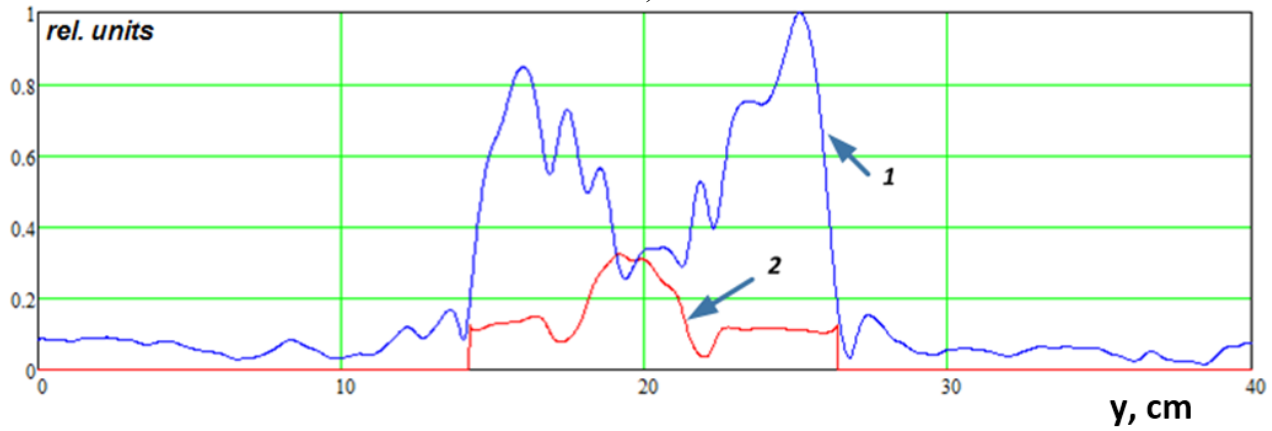

b)

Figure 10. Intensity and phase distribution along metal plate and along metal plate with dielectric brick.

\section{Conclusion}

Experiments made during research are the proof of relief restoration of non-flat objects by monochrome radiowave radiation. Problem with distance definition can be solved by focusing after multipositional scanning. Phase information of received signal allows restoring relief of scanned object. Proposed methods make possible to use monochrome radiation for radioimage restoration. Using of monochrome sensors as part of scanning systems is possible way to reduce such systems' costs while keeping image quality at the same level.

\section{Acknowledgment}

The work is done as part of the State Assignment No. 3.694.2014/K of the Russian Ministry of Education and Science.

\section{References}

[1] A. Bennamane, T. Boutkedjirt, MATEC Web of Conferences 16, 10002 (2014) doi: $10.1051 /$ matecconf $/ 20141610002$

[2] R. Appleby, Philosophical Transactions: Mathematical, Physical and Engineering Sciences 362, 379 (2004) doi: 10.1098/rsta.2003.1323

[3] R. N. Satarov, I. Yu. Kuz'menko, T. R. Muksunov, A. V. Klokov, E. V. Balzovskii, Yu. I. Buyanov, S. É. Shipilov, V. P. Yakubov, Russian Physics Journal 55, 884 (2013) doi: 10.1007/s11182-013-9896-8 
[4] J.E. Bjarnason, T. L. J. Chan, A. W. M. Lee, M. A. Celis, E. R. Brown, Applied Physics Letters 85, 519 (2004) doi: 10.1063/1.1771814

[5] D.Y. Sukhanov, K.V. Zavyalova, Technical Physics 57, 819 (2012) doi: $10.1134 / \mathrm{S} 1063784212060229$

[6] F.-G. Joaquim, IEEE Transactions on Geosciences and Remote Sensing 40, 443 (2002) doi: 10.1109/36.992808

[7] J. Groenenboom, A.G. Yarovoy, GPR 2000: Proceedings of the eighth international $\begin{array}{llll}\text { conference on ground penetrating radar 4084, } 867 & \text { (2000) }\end{array}$ doi: $10.1117 / 12.383530$ 